\title{
Flow-induced voltage and current generation in carbon nanotubes
}

\author{
S. Ghosh ${ }^{1}$, A.K. Sood ${ }^{1}$, S. Ramaswamy ${ }^{1}$ and N. Kumar ${ }^{2}$ \\ ${ }^{1}$ Department of Physics, Indian Institute of Science, Bangalore - 560 012, India and \\ ${ }^{2}$ Raman Research Institute, C.V. Raman Avenue,Bangalore - 560 080, India
}

\begin{abstract}
New experimental results, and a plausible theoretical understanding thereof, are presented for the flow-induced currents and voltages observed in single-walled carbon nanotube samples. In our experiments, the electrical response was found to be sublinear - nearly logarithmic - in the flow speed over a wide range, and its direction could be controlled by an electrochemical biasing of the nanotubes. These experimental findings are inconsistent with the conventional idea of a streaming potential as the efficient cause. Here we present Langevin-equation based treatment of the nanotube charge carriers, assumed to be moving in the fluctuating field of ions in the flowing liquid. The resulting "Doppler-shifted" force-force correlation, as seen by the charge carriers drifting in the nanotube, is shown to give a sublinear response, broadly in agreement with experiments.
\end{abstract}


Single-walled carbon nanotubes (SWNT) in contact with a flowing liquid provide a unique microfluidic system that offers a large interfacial area of intimate atomic contact between the liquid and the solid substrate. This can lead to a strong coupling of the charge carriers in the nanotube to the particles in the flowing liquid, more so if the liquid is polar or ionic in character. The effect of this coupling is expected to be further enhanced due to charge carrier entrainment because of the quasi-one dimensionality of the conducting nanotubes. Recently, the flow of a variety of liquids over SWNT bundles was studied experimentally, and was found to generate voltage in the sample along the direction of the flow [1]. Quite unexpectedly, however, the dependence of the voltage on the flow speed was found to be sublinear, and could be fitted to a logarithmic form over five decades of variation of the speed. There has been an attempt at explaining away this flow-induced voltage in electrokinetic terms as the streaming potential that develops along the flow of an electrolyte through a microporous insulator 2]. Earlier Král and Shapiro 3] proposed that the shear stress of liquid flow transfers momentum to the acoustic phonons of the nanotube and the resulting "phonon wind" produces a current of carriers in the nanotube. They also suggested, qualitatively, that the fluctuating Coulomb fields of the ions in the liquid could drag directly the carriers in the nanotubes. To produce a flow-rate of $1 \mathrm{~cm} / \mathrm{s}$ in an inter-nanotube channel of 20nm width, as demanded by the first mechanism [3] requires an enormous pressure head, about $10^{13}$ dynes $/ \mathrm{cm}^{2}$. The second mechanism [3] gives currents of order femtoAmperes. In both mechanisms [3], the effect is linear in the flow rate, in contrast to the experimental findings [1]. The mechanism we offer is related to the second idea of [3] but requires neither localization of carriers nor drag at the same speed as the ions. The main aim of this paper is to report (i) our new experimental results of the measurements of the the short-circuit electrical current as a function of the flow-speed, (ii) ways to control the magnitude and directionality of the flow induced current; and (iii) a general theory for the electrical response consistent with these new observations. Our measurements clearly rule out the electrokinetic mechanism based on the idea of a streaming potential. Our theory is based on a stochastic treatment of the nanotube charge-carriers assumed to be moving under the influence of the correlated ionic fluctuations which are advected by the liquid flow. It is broadly consistent with our experiments.

Figure 1 shows a schematic sketch of the construction of the flow sensor. SWNT bundles prepared by arc discharge method [4] are packed between two metal electrodes. The nan- 
otubes are kept in their place by a supporting insulating substrate. The electrical signal is measured along the flow direction $\left(u_{L}\right)$ as shown Fig. 1. The other experimental details are as in [1]. A sensor with a minimal contact resistance of $\sim 25 \Omega$ (found from four- probe measurements) was used in the experiments so that the short-circuit current could be measured. The short-circuit current (open-circuit voltage) was measured by connecting the microammeter (millivoltmeter) across the SWNT sample. The resistance (two-probe) of the device, measured with the sensor dipped in the liquid was found to be $\sim 70 \Omega$. Figure 2 shows the dependence of the induced voltage and current on the flow velocity $\mathrm{u}_{L}$. The solid line is a fit to the empirical relation $I=\alpha_{I} \log \left(\beta_{I} u_{L}+1\right)$, with $\alpha_{I}=0.02 \mu A$ and $\beta_{I}=4.8 \times 10^{4} \mathrm{~s} / \mathrm{cm}$. The voltage also fits the empirical relation $V=\alpha_{V} \log \left(\beta_{V} u_{L}+1\right)$, where $\alpha_{V}=1.4 \mu V$ and $\beta_{V}=4.8 \times 10^{4} \mathrm{~s} / \mathrm{cm}$. It can readily be seen that $\alpha_{V}=\alpha_{I} \times R$, i.e., the resistance encountered is precisely the resistance ( 2 probe) of the device. This is an important point to note: if an electrokinetic mechanism were operating, the resistance obtained would have been orders of magnitude higher, i.e., equal to that of the electrolyte $(\sim 0.1 M \Omega)[\underline{5]}$. This in itself rules out quite decisively the electrokinetic mechanism of voltage generation. Next, we consider the measured direction of the flow induced current with respect to the flow direction as a function of the bias voltage $V_{B}$ (see inset of Fig 3). This potential biases the SWNT with respect to the Au-reference electrode immersed in the flow chamber close to sample as shown in the inset of Fig.(3). The dependence of the sign and the magnitude of the flow-induced voltage on $V_{B}$ for an aqueous solution of $0.01 \mathrm{M} \mathrm{KCl} \mathrm{(conductivity} 1.4 \mathrm{mS} / \mathrm{m}$ ) and for a fixed flow speed of $0.04 \mathrm{~cm} / \mathrm{s}$ is shown in Fig.(3). It is seen that the flow-induced signal is positive, i.e., $I$ is anti-parallel to $u_{L}$ when $V_{B}$ is positive, and the sign of the signal is reversed, i.e., $I$ is parallel to $u_{L}$, for $V_{B}$ negative, consistent with our theory. Further, in our theoretical mechanism, based on fluctuations, the carriers in the nanotube drift necessarily in the same direction as the flow velocity $u_{L}$, quite independently of the sign of the ionic charge. Thus, for the current $I$ to be parallel (antiparallel) to $u_{L}$ the charge carriers in the nanotubes need to be holes (electrons). When the nanotubes are biased positively, the anions $\left(\mathrm{Cl}^{-}, \mathrm{OH}^{-}\right)$ move closer to the SWNT, localizing holes on the carbon and making electrons available for flow-induced current. Similarly, holes are liberated when the bias is negative [6]. As the bias voltage is increased the number of carriers participating in the flow-induced current will increase as shown in Fig.(3). Thus, the results obtained by electrochemically biasing the nanotubes are naturally consistent with our mechanism of (coulombic) forcing of the 
nanotube charge carriers by the liquid flow. The dependence of the flow-induced signal on the concentration of different types of ions in the liquid is, however, found to be complicated and non-monotonic. These details will not be addressed here.

We now turn to our theoretical mechanism for the flow-induced current/voltage. This can be understood qualitatively in terms of three physically distinct but related ideas: (a) Induced friction: The fluctuating charge density of the ions close to the nanotube couples couloumbically to the charge carriers in the nanotube and, therefore, offers a friction to the motion of these charge carriers (in addition to the Ohmic friction intrinsic to the carbon nanotubes). This, of course, follows directly from the fluctuation-dissipation theorem; (b) Flow-induced drag: In virtue of the above frictional coupling, an imposed liquid flow drags the charge carriers along through the nanotube; (c) Reduction of induced friction at high flow speeds: The space-time correlated couloumbic fluctuations, inherent to the liquid electrolyte, are advected by the liquid flow, and thus get Galilean boosted (Doppler shifted) as seen in the mean rest frame of the drifting carriers in the nanotube. Correspondingly, as we will see, the friction they offer to the motion of the charge carriers in the nanotube diminishes with increasing flow speed. This is crucial to the observed sublinear dependence of the charge drift-velocity (electrical response) on the liquid flow speed.With the above in mind, we will now derive these frictional effects, first from a heuristic argument, and then analytically from a Langevin-equation treatment.

Consider a nanotube placed along the the $z$-axis with the liquid flowing parallel to it. We model the nanotube as a classical one- dimensional (1D) conductor with diffusive charge transport. In the steady state, let $u_{L}$ and $u_{D}$ be, respectively, the velocity of liquid(L) flow and the induced drift velocity of charge carriers in the nanotubes, all measured relative to the nanotube lattice. Now, in the absence of any frictional coupling to the liquid flow, the drift velocity $u_{D}$ will tend to relax to zero, i.e., to rest with respect to the lattice, with a relaxation time $\tau_{D}$ characteristic of the nanotube resistivity. Similarly, if we were to "switch off" the resistive coupling to the lattice, the drift velocity $u_{D}$ would relax to the liquid velocity $u_{L}$, i.e., to the rest frame co-moving with the liquid, because of the frictional coupling (drag), with a relaxation time $\tau_{L}$. Now, therefore, in the presence of both these frictional influences, the drift velocity $u_{D}$ will assume a steady-state value $u_{D}<u_{L}$ that 
satisfies $u_{D} / \tau_{D}=\left(u_{L}-u_{D}\right) / \tau_{L}$ giving

$$
u_{D}=u_{L} /\left(1+\tau_{L} / \tau_{D}\right)
$$

Equation (11) is merely a restatement of the condition of frictional force-balance in the steady state. It would appear to give an induced short-circuit current (equivalently, an open circuit voltage via the nanotube resistance) along the nanotube which is linear in the flow velocity. The nonlinearity is, however, really hidden in the $u_{L}$ dependence of the relaxation time $\tau_{L}$ that we will now try to make explicit. It may be noted here that we are assuming, for simplicity, a uniform liquid flow without the hydrodynamic complications of a no-slip boundary condition.

In a simple caricature of the real situation then, consider the ionic density in the liquid, fluctuating thermally and flowing past the nanotube at a mean velocity $u_{L} \hat{\mathbf{z}}$, producing thereby a fluctuating couloumbic potential $\phi(\mathbf{r}, t)$, at a point $\mathbf{r}$ at time $t$. We are, of course, interested in the case of $\mathbf{r}$ lying on the $z$ axis i.e., $\mathbf{r}=(0,0, z)$ (in the $1 \mathrm{D}$ nanotube). For the space-time correlation function $\langle\phi(0,0) \phi(\mathbf{r}, t)\rangle \equiv G_{0}(\mathbf{r}, t)$ in the mean rest frame of the ions, the charge carriers in the nanotube see this correlation Galilean boosted to $G(\mathbf{r}, t) \equiv G_{0}(\mathbf{r}-\hat{\mathbf{z}} v t, t)$ with $v=u_{L}-u_{D}$. This Galilean boost (Doppler shift) is the key physical point of our treatment. At $u_{L}=0$, the fluctuation-dissipation theorem (FDT) tells us that the coefficient of the zero-frequency friction to the motion of the charge carriers in the nanotubes, arising from the ionic thermal fluctuations, is proportional to the time integral of this on-site force-force correlation function. If we assume this relation even for $u_{L} \neq 0$ we have

$$
1 / \tau_{L}=1 /\left(m_{e} k_{B} T\right) \int_{-\infty}^{\infty}\left\langle e E_{z}(\mathbf{r}-\hat{\mathbf{z}} v t, t) e E_{z}(r, 0)\right\rangle d t
$$

Here $E_{z}$ is the $z$-component of the coulombic (electric ) field due to the ions; $m_{e}$ is the mass of the charge carrier with $e$ the electronic charge; $k_{B}$ is the Boltzman constant, and $T$ the absolute temperature. We re-write the right hand side of Eqn.(2) in Fourier (q)- space, expressing the above force-force correlator in terms of the ionic charge-densities $\rho(\mathbf{r}, t)$ using $E_{z}(\mathbf{q}, t)=-i q_{z} \phi(\mathbf{q}, t)$ and $-q^{2} \phi(\mathbf{q})=e \rho(\mathbf{q}, t) / \epsilon$, where $\epsilon$ is the solvent dielectric constant, and obtain straightforwardly

$$
\begin{aligned}
\frac{1}{\tau_{L}} & =\frac{1}{m_{e} k_{B} T}\left(\frac{4 \pi e^{2}}{\epsilon}\right)^{2} \rho_{0} \int \frac{d \mathbf{q}}{(2 \pi)^{3}}\left(\frac{q_{z}^{2}}{q^{4}}\right)\left(\frac{q^{2}}{q^{2}+\kappa^{2}}\right)\left(\frac{2 / \tau_{q}}{\left(v q_{z}\right)^{2}+\left(1 / \tau_{q}\right)^{2}}\right) \\
& =\frac{\rho_{0}}{4 \pi m_{e} D \kappa k_{B} T}\left(\frac{4 \pi e^{2}}{\epsilon}\right)^{2} \frac{1}{x}\left(1-\frac{2}{x}+\frac{1}{x^{2}} \log \left|1-x^{2}\right|-\frac{1}{x^{2}} \log \left|\frac{1-x}{1+x}\right|\right)
\end{aligned}
$$


where $x=v / D \kappa$, and $\rho_{0}$ is the mean ionic number density. In Eqns. (3) and (44) we have used the Debye -screened form for the static charge structure factor $S_{q}^{0}=\left\langle\rho_{q} \rho_{-q}\right\rangle=$ $q^{2} /\left(q^{2}+\kappa^{2}\right)$ with screening length $\kappa^{-1}$ as the inverse of the Debye screening length and a diffusive form $1 / \tau_{q}=D q^{2}[$ [] with $D$ the ionic diffusion constant. It can be seen at once that Eqn.(44), taken in conjunction with Eqn.(2), gives a drift velocity $u_{D}$ (and therefore the short-circuit current) as a sublinear function of the flow velocity $u_{L}$. This sublinearity is a generic feature of this mechanism, and is clearly seen in inset of Fig.(2) in which we have plotted the induced current versus the liquid flow speed derived directly from Eqn.(4) for certain choice of parameters. Rather than attempting to justify this use of the regression formula in a nonequilibrium context, we turn instead to a direct evaluation of the drift speed starting from a Langevin equation. We recover the same sublinear functional form for the response as in Eqn.(3).

The one-dimensional (1D) position $z(t)$ of a tagged charge carrier in the nanotube obeys the overdamped Langevin equation

$$
\zeta \frac{d z}{d t}=F(z(t), t)+f(t)
$$

where $F(z(t), t)$ is the fluctuating force the ions exert on the carrier at $z(t)$ in the nanotube, and $f$ and $\zeta$ are the thermal Gaussian white noise and friction intrinsic to the nanotube, with $\langle f(0) f(t)\rangle=2 k_{B} T \zeta \delta(t)$. Strictly speaking, $F(z(t), t)$ should be a function of the instantaneous separations between the positions $z(t)$ of the tagged carriers in the nanotube and those of all the ions in the ambient liquid. We would like to replace this complex manybody problem by an effective Langevin equation for the dissipative motion of the charge carriers in the nanotube, without invoking the FDT which, strictly speaking, holds only in the absence of the flow. Our treatment nonetheless captures the qualitative physics of the dissipative entrainment of carriers by the moving ions. For this, we simply take $F(z, t)$ to be a Gaussian random force field, with zero mean and a two-point correlation $C(z, t)$. Then from Eqn.(5), the drift velocity of the carrier $u_{D} \equiv\langle d z(t) / d t\rangle=(1 / \zeta)\langle F(z(t), t)\rangle$ can be rewritten, using Novikov's theorem [8] in relation to the Gaussian noise $F(z, t)$, as

$$
\begin{aligned}
u_{D} & =\frac{1}{\zeta} \int_{z, z^{\prime}, t^{\prime}} C\left(z-z^{\prime}, t-t^{\prime}\right)\left\langle\frac{\delta \delta(z-z(t))}{\delta F\left(z^{\prime}, t^{\prime}\right)}\right\rangle \\
& =-\frac{1}{\zeta} \int_{z, z^{\prime}, t^{\prime}} C\left(z-z^{\prime}, t-t^{\prime}\right)\left\langle\frac{\delta z(t)}{\delta F\left(z^{\prime}, t^{\prime}\right)} \delta^{\prime}(z-z(t))\right\rangle .
\end{aligned}
$$

Using Eqn.(5) to evaluate the functional derivative in Eqn.(6), and writing $C(z, t)$ in terms 
of its Fourier transform $C_{q}(t)$ yields

$$
u_{D}=\frac{1}{\zeta^{2}} \int_{-\Lambda}^{\Lambda} \frac{\mathrm{d} \mathbf{q}}{(2 \pi)^{3}} \int_{0}^{\infty} \mathrm{d} t\left\langle e^{i q_{z}\left[z(t)-z\left(t^{\prime}\right)\right]}\right\rangle i q_{z} C_{q}(t)
$$

where $\Lambda$ is an ultraviolet cut-off of the order of an inverse ionic diameter. As in the preceding heuristic treatment, let us take the correlation $C_{q}(t)$ of the ions to be the Galilean boost, with velocity $u_{L}$, of an equilibrium correlation function $C_{q}^{0}(t)$, with a relaxation time $\tau_{q}:$

$$
C_{q}(t)=C_{q}^{0}(t) e^{-i q_{z} u_{L} t} \equiv C_{q}^{0} e^{-i q_{z} u_{L} t} e^{-t / \tau_{q}},
$$

where $C_{q}^{0}$ is the equilibrium equal-time correlation function of the force fluctuations. This form, despite its undeniable limitations, is the simplest way to capture the basic physics of ions moving past the nanotube, and admits an essentially analytical treatment. As before, the force-force correlation $C_{q}^{0}$ can be expressed in terms of the ionic charge-density correlation, which is known as an input from the liquid state(dilute ionic solution) theory, namely that $C_{q}^{0} \propto\left(q_{z}^{2} / q^{4}\right) S_{q}^{0}$ with the ionic charge structure factor $S_{q}^{0}=q^{2} /\left(q^{2}+\kappa^{2}\right)$. Note the factor $\left(q_{z}^{2} / q^{4}\right)$ arising from the gradient $(\partial / \partial z)$ and the Laplacian $\left(\nabla^{2}\right)$ in $q$ - space. Replacing $z(t)$ in Eqn.(17) by its mean $u_{D} t$ for simplicity, we obtain the compact expression

$$
u_{D}=v \alpha \int_{-\Lambda}^{\Lambda} \frac{\mathrm{d} \mathbf{q}}{(2 \pi)^{3}}\left(\frac{1}{q_{z}^{2} v^{2}+\tau_{q}^{-2}}\right)\left(\frac{q_{z}^{2}}{q^{2}+\kappa^{2}}\right),
$$

for the drift velocity of the charge carriers in the nanotube, where as before $v=u_{L}-u_{D}, \alpha$ is a lumped constant of proportionality that depends on the parameters of the liquid-state correlation function input used above. With the ultraviolet cut-off $(\Lambda)$ set to infinity, and [7] with $1 / \tau_{q}=D q^{2}$, Eqn. (9) has precisely the form of Eqn.(11) taken in conjunction with the Eqn.(4), allowing us thereby to identify the integral on its right-hand side essentially with $\tau_{D} / \tau_{L}$. This gives us an expression for the flow-speed dependence of $\tau_{L}$, and thus finally an analytic expression for the charge drift velocity $\left(u_{D}\right)$ as a function of the liquid flow velocity $\left(u_{L}\right)$. This reaffirms our heuristic argument given at the beginning.

We close by summarizing the main points of our work. First, on the experimental side, we have clearly shown that the liquid flow produces not only a voltage (i.e., not merely a capacitive charging), but a short-circuit current as well in the nanotube; that both have a sublinear dependence on the imposed flow-speed; and that the voltage/current ratio corresponds to the nanotube sample resistance. These observations are incompatible with an electrokinetic origin for the (electronic) current in the nanotube. On the theoretical side, 
we have proposed a theory wherein the current is essentially a statistical consequence of the flow-induced asymmetry in the correlation of the ions, in the ambient fluid as seen by the charge carriers in the nanotube. Importantly, our theory predicts in general a sublinear behavior for the electrical response, with a linear regime at only the smallest values of imposed flow. The extended logarithmic regime seen in experiments can presumably be rationalized in detail with particular forms for the correlation function $\left(C_{q}^{0}\right)$ and the relaxation time $\left(\tau_{q}\right)$, as inputs to be taken from the liquid state (dilute ionic solution) theory. Moreover, a realistic treatment will require taking into account details of the complex, hydrophobic, inter-nanotube micro-fluidic environment of our mat samples. Thus, very specifically, the no-slip boundary condition would imply a decreasing velocity of the flow nearer the nanotube (the shear flow).This decrease in the flow velocity will,however,be offset by the corresponding increase in its effectiveness(via the screened coulombic forcing) closer to the interface.The resulting levelling is expected to broaden the sublinear response and thus improve agreement with the experiment. Our main point, however, is that the experiments show sublinear behavior, which is inconsistent within existing theories of flow-induced voltages and currents in nanotubes, and that our approach naturally and inevitably leads to strong sublinearity. Finally, we emphasize that the flow-induced asymmetry of the random fluctuations is key to the charge-carrier drift (drag) mechanism in our theory. In this broad sense our approach here subsumes the asymmetric fluctuating ratchets invoked earlier [1] in a general way. In this connection, reference must be made to the idea of a drag (shear) induced by the relative motion between material surfaces, where the Doppler shifted and aberrated photonic fluctuations, e.g. zero-point photons, have been invoked very effectively [9, 10]. We would also like here to add that more than one mechanisms could very well be at work in these systems. For example, in a recent publication Persson et al 11] have invoked a combination of frictional stick-slip and barrier-hopping to explain the observed phenomenon of flow-induced voltages in SWNT. The one we propose here seems particularly robust and general, and we look forward to experimental tests, especially of the predicted saturation of the electrical response at high flow speeds.

SR (through the Center for Condensed Matter Theory) and AKS thank the DST, India, for support. AKS thanks Prof. C.N.R. Rao for nanotube samples and fruitful collaboration on nanotubes. 
[1] S. Ghosh, A.K. Sood and N. Kumar, Science 299, 1042 (2003).

[2] A. E. Cohen (comment); S. Ghosh, A. K. Sood, and N. Kumar (reply); Science 300, 1235 (2003).

[3] P.Král and M.Shapiro, Phys.Rev.Lett. 86, 131 (2001).

[4] P. V. Teredesai, A.K. Sood, D. V. S. Muthu, R. Sen, A. Govindraj C. N. R. Rao, Chem. Phys. Lett., 319, 296 (2000).

[5] S.Glasstone, An Introduction to Electrochemistry, Affiliated, East West Press, New Delhi, (reprinted 2003);Ch. 16.

[6] S. Kazaoui and N. Minami and N. Matsuda and H. Kataura and Y. Achiba, Appl. Phys. Lett 78, 3433 (2001).

[7] Consistency demands $\tau_{q}=S_{q}^{0} / D q^{2}$; we have checked numerically that this form also produces qualitatively the same shape as in the inset to Fig.(1).

[8] E. A. Novikov, Zh. Eksp. Teor. Fiz. 47,1919(1964), Sov. Phys. JETP 20, 1990 (1969)

[9] J.B. Pendry, J. Phys: Condens Matter 9, 10301 (1997).

[10] P. M. Solomon, P. Price, D. Frank and D. L. Tulipe Phys. Rev. Lett. 63, 2508 (1989).

[11] B. N. J. Persson, U. Tartaglino, E. Tosatti, and H. Ueba, Phys. Rev. B 69, 235410(2004) 


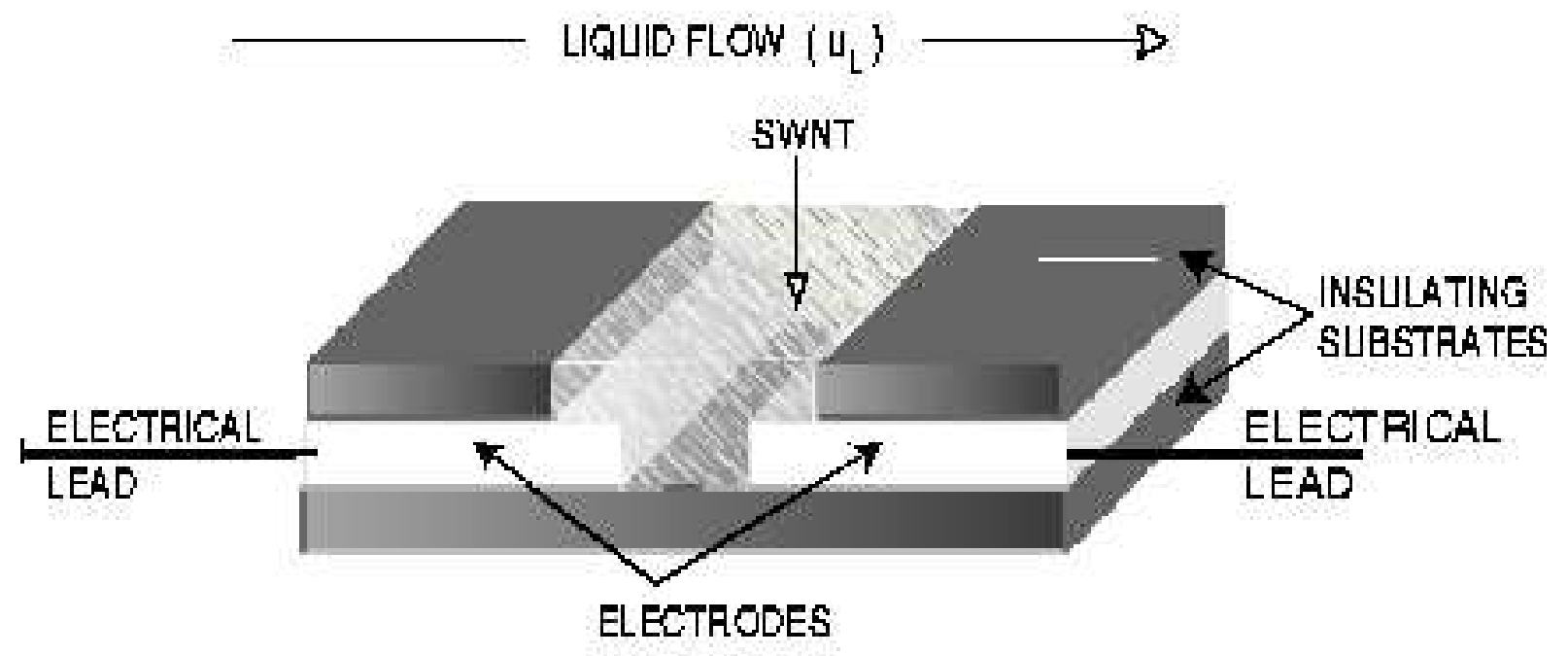

FIG. 1: Schematic sketch of the nanotube flow sensor placed along the flow $\operatorname{direction}\left(u_{L}\right)$. SWNT bundles are packed between two metal electrodes. The insulating substrate keeps the SWNT in place. The electrical leads are taken out from the metal electrodes. 


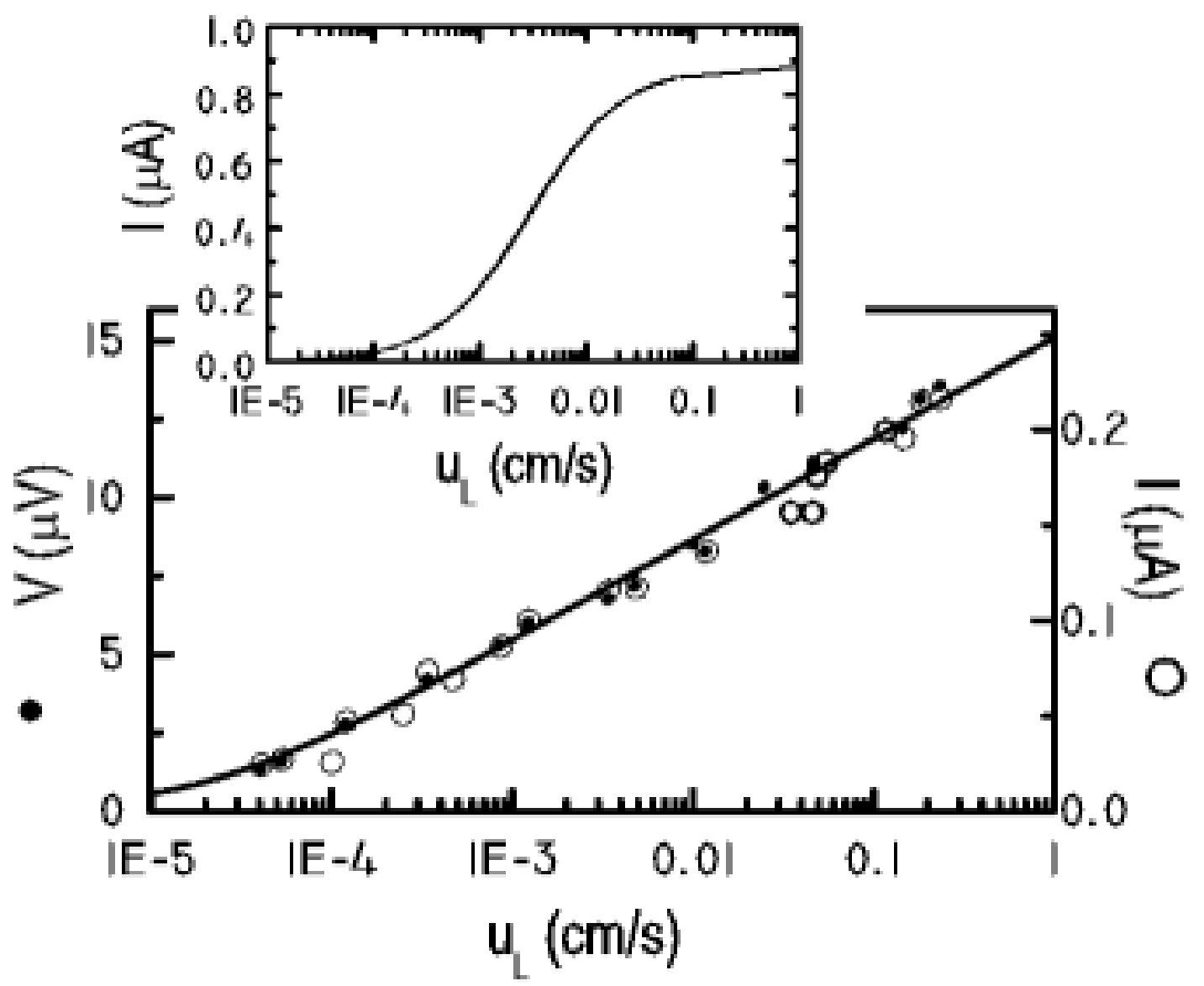

FIG. 2: Voltage (full circles) and current (open circles) as functions of flow speed $u_{L}$. The solid line is a fit to the logarithmic function as explained in the text. Inset shows the theoretical plot of current $\left(I=n e u_{D} A\right)$ versus flow speed based on Eqn.(14) for typical choice of parameters: $\mathrm{T}=300 \mathrm{~K} ; \epsilon=80$ ( CGS units); $D \kappa=10^{-4} \mathrm{~cm} / \mathrm{s} ; \tau_{D}=10^{-16} \mathrm{~s} ; \rho_{0}=10^{13} \mathrm{~cm}^{-3}$; charge carrier density in nanotubes $\left(n=10^{18} \mathrm{~cm}^{-3}\right)$; cross-sectional-area $(A)=10^{-3} \mathrm{~cm}^{2}$. The strong sublinearity is clearly seen. 


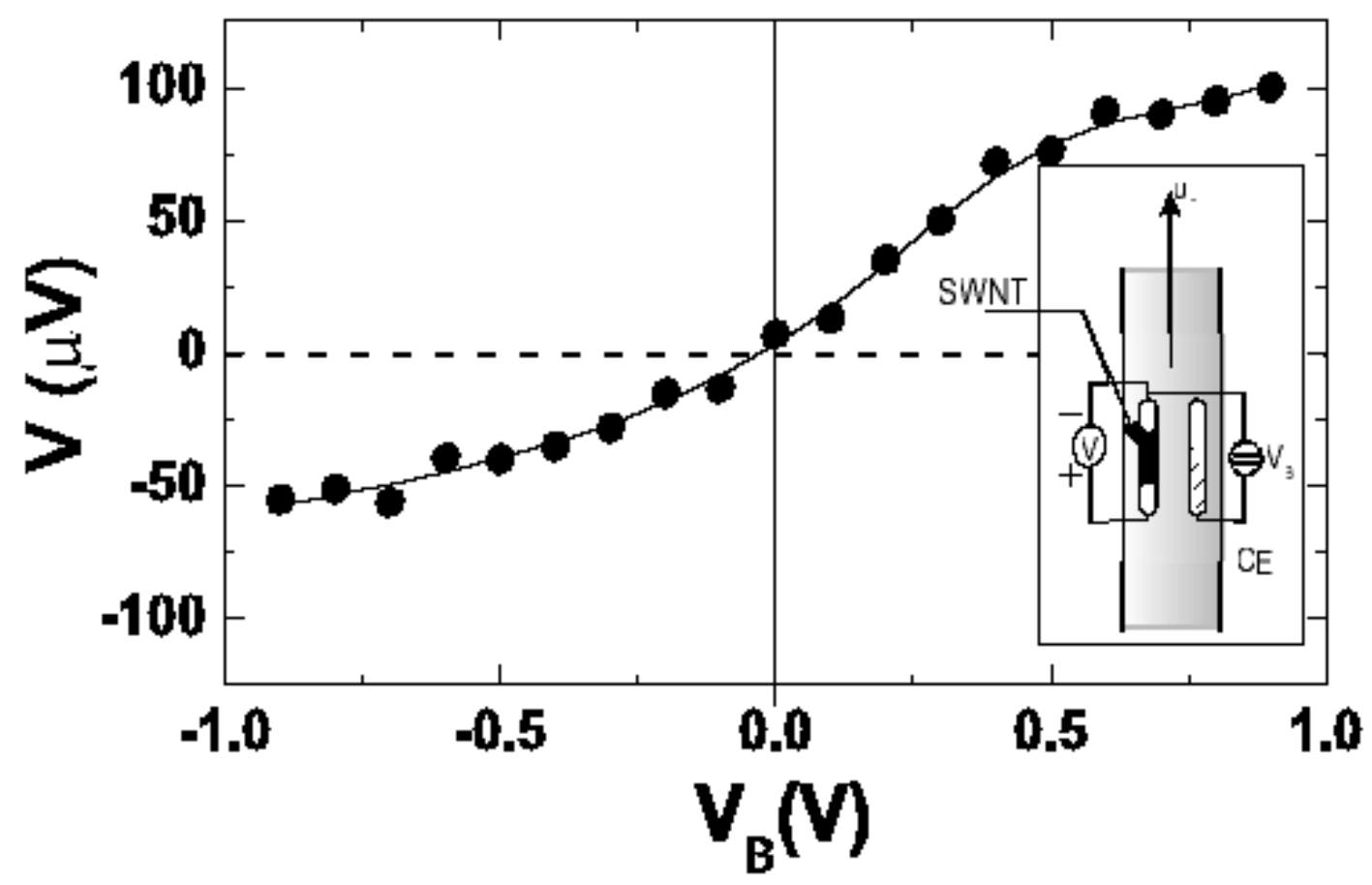

FIG. 3: Flow-induced voltage as a function of bias $V_{B}$. Inset: schematic of electrochemical biasing of the nanotubes; $\mathrm{CE}$ is the counterelectrode.The solid line is a guide to the eye 